\title{
Evaluation of the nephrotoxicity and safety of low- dose aristolochic acid, extending to the use of Xixin (Asurum), by determination of methylglyoxal and D- lactate
}

Chia-En Lin

Taipei Medical University

Po-Yeh Lin

Taipei Medical University

Wen-Chi Yang

Taipei Medical University

Yu-Shen Huang

Taipei Medical University

Tzu-Yao Lin

Taipei Medical University

ChienखMing Chen

National Taipei University of Technology

Hung-Shing Chen

National Taiwan University of Science and Technology

Jen-Ai Lee

Taipei Medical University

Shih-Ming Chen ( $\nabla$ smchen@tmu.edu.tw )

Taipei Medical University College of Pharmacy https://orcid.org/0000-0002-0273-4847

Research

Keywords: Aristolochic acid (AA), D-lactate, fibrosis, methylglyoxal, nephrotoxicity

Posted Date: July 29th, 2020

DOI: https://doi.org/10.21203/rs.3.rs-48442/v1

License: (c) (1) This work is licensed under a Creative Commons Attribution 4.0 International License.

Read Full License 
Version of Record: A version of this preprint was published at Journal of Ethnopharmacology on May 1st, 2021. See the published version at https://doi.org/10.1016/j.jep.2021.113945. 
1 Evaluation of the nephrotoxicity and safety of low-dose aristolochic acid, extending to

2 the use of Xixin (Asurum), by determination of methylglyoxal and D-lactate

3

4 Chia-En Lin ${ }^{\mathrm{a}}$, Po-Yeh Lin ${ }^{\mathrm{a}}$, Wen-Chi Yang ${ }^{\mathrm{a}}$, Yu-Shen Huang ${ }^{\mathrm{a}}$, Tzu-Yao Lin ${ }^{\mathrm{a}}$, Chien-

5 Ming Chen ${ }^{\mathrm{b}}$, Hung-Shing Chen ${ }^{\mathrm{c}}$, Jen-Ai Lee ${ }^{\mathrm{a}, *}$, Shih-Ming Chen ${ }^{\mathrm{a},{ }^{*}}$

6

$7 \quad{ }^{a}$ School of Pharmacy, College of Pharmacy, Taipei Medical University, No.250 Wu-

8 Hsing Street, Taipei 110, Taiwan

$9{ }^{\mathrm{b}}$ Department of Electro-Optical Engineering, National Taipei University of

10 Technology, Taipei, Taiwan

$11{ }^{\mathrm{c}}$ Graduate Institute of Electro-optical Engineering, National Taiwan University of

12 Science and Technology, No.43, Keelung Rd., Sec.4, Da'an Dist., Taipei, Taiwan

$14{ }^{*}$ Corresponding author. Tel./fax: +886-2-2736-1661 ext.6176.

15 E-mail address: smchen@tmu.edu.tw (Shih-Ming Chen).

16 *Additional corresponding author. Tel./fax: +886-2-2736-1661 ext.6125.

17 E-mail address: jenai@tmu.edu.tw (Jen-Ai Lee).

18 Co-author E-mail: Chia-En Lin (d301106002@tmu.edu.tw) ; Po-Yeh Lin

19 (poyeh0111@gmail.com); Wen-Chi Yang (gigyang311@gmail.com); Yu-Shen 
21 Ming Chen (cmchen@ mail.ntut.edu.tw); Hung-Shing Chen

22 (bridge@mail.ntust.edu.tw).

24 Abstract

25 Background: Most Aristolochiaceae plants are prohibited due to aristolochic acid nephropathy (AAN), except Xixin (Asarum spp.). Xixin contains trace amounts of

27 aristolochic acid (AA) and is widely used in Traditional Chinese Medicine.

28 Methylglyoxal and D-lactate are regarded as biomarkers for nephrotoxicity. Thus, this

29 study aimed to evaluate tubulointerstitial injury and interstitial renal fibrosis by

30 determining urinary methylglyoxal and D-lactate after withdrawal of low-dose AA in

31 a chronic mouse model.

32 Methods: $\mathrm{C} 3 \mathrm{H} / \mathrm{He}$ mice in the AA group ( $n=24 /$ group) were given ad libitum access

33 to distilled water containing $3 \mu \mathrm{g} / \mathrm{mL} \mathrm{AA}(0.5 \mathrm{mg} / \mathrm{kg} /$ day $)$ for 56 days and drinking

34 water from days 57 to 84 . The severity of tubulointerstitial injury and fibrosis were

35 evaluated using the tubulointerstitial histological score (TIHS) and Masson's

36 trichrome staining. Urinary and serum methylglyoxal were determined by high-

37 performance liquid chromatography (HPLC); urinary D-lactate were determined by

38 column-switching HPLC. 
39 Results: After AA withdrawal, serum methylglyoxal in the AA group increased from day $56(429.4 \pm 48.3 \mu \mathrm{g} / \mathrm{L})$ to $84(600.2 \pm 99.9 \mu \mathrm{g} / \mathrm{L})$, and peaked on day $70(878.3 \pm$

$41 \quad 171.8 \mu \mathrm{g} / \mathrm{L} ; p<0.05)$; TIHS and fibrosis exhibited similar patterns. Urinary methylglyoxal was high on day $56(3.522 \pm 1.061 \mu \mathrm{g})$, declined by day $70(1.583 \pm$ $0.437 \mu \mathrm{g})$ and increased by day $84(2.390 \pm 0.130 \mu \mathrm{g})$. Moreover, urinary D-lactate was elevated on day $56(82.10 \pm 18.80 \mu \mathrm{g})$ and higher from day $70(201.10 \pm 90.82 \mu \mathrm{g})$ to $84(193.28 \pm 61.32 \mu \mathrm{g})$.

Conclusions: Methylglyoxal is induced after AA-induced tubulointerstitial injury, thus methylglyoxal excretion and metabolism may be a detoxification and repair strategy. A low cumulative AA dose is the key factor that limits tubulointerstitial injury and repair. Thus, AA-containing herbs, especially Xixin, should be used at low doses for short durations (less than one month).

51 Keywords: Aristolochic acid (AA), D-lactate, fibrosis, methylglyoxal, nephrotoxicity

\section{Background} Aristolochiaceae plants are traditionally used in several Traditional Chinese

55 Medicine (TCM) formulas, such as Xiao-Qing-Long-Tang, Chuan Xiong Cha Tiao

56 San, Ma Huang Fu Zi Xi Xin Tang, Qing Shang Juan Tong Tang and Du Hwa Jih

57 Sheng Tang. However, as they induce aristolochic acid nephropathy (AAN), most 
58 aristolochic acid (AA)-containing plants is prohibited, including Aristolochia contorta

59 (Ma Dou Ling) [1], Aristolochia fangchi (Guang Fang Ji) [1], Aristolochia

60

61

62 manshuriensis (Guan Mu Tong) [1], Aristolochia contorta (Tian Xian Teng) and Aristolochia debilis (Qing Mu Xiang) [1]. These species can be replaced in Traditional Chinese Medicine (TCM) by other non-Aristolochiaceae plants [2]. However, Xixin cannot be replaced by other non-Aristolochiaceae plants. All origins of Xixin, including Asarum heterotropoides Fr. Schmidt var. mandshuricum (Maxim.) Kitag, Asarum crispulatum C.Y. Cheng and C.S. Yang, Asarum forbesii Maxim, A. himalaicum Hooh. F. and Thoms. Ex Klotzsch, Asarum sieboldii Miq, Asarum debile Franch Asarum maximum Hemsl, Asarum ichangense C.Y. Cheng and C.S. Yang, and Asarum fukienense C.Y. Cheng and C.S. Yang, are members of the Aristolochiaceae family. Moreover, Xixin and its combination with other herbs are frequently used to treat a variety of conditions, including fever [3], influenza in the elderly [4], infection [5], allergy [6], caries [7], inflammation [8, 9], pain [8], rhinitis [9] and rheumatoid arthritis [10]. Consequently, while the use of AA-related products, especially Xixin, remains essential, their safety is controversial.

4 Methylglyoxal and D-lactate can be used to assess nephrotoxicity and play key 5 roles in the progression of renal injury, including diabetic [11], gentamicin-induced [12], Pb-induced [13] and acute AA-induced nephropathy [14-16]. The highly reactive 
77 dicarbonyl groups of methylglyoxal denature proteins and nucleic acids; the resulting

78 methylglyoxal-derived compounds are called advanced glycation end products

79 (AGEs) [17]. As a mechanism to limit the toxicity of methylglyoxal, methylglyoxal is

80 metabolized into D-lactate via the glyoxalase system $[18,19]$. Thus, D-lactate is

81 considered to be a marker of renal damage and accumulation of methylglyoxal. On

82 the other hand, renal injury increases both D-lactate and L-lactate, while prednisolone

83 [18] or metformin [10] treatment alleviate histological damage and decrease D-lactate

84 - rather than L-lactate or D/L-lactate - in AAN models. Nevertheless, the changes in

85 methylglyoxal and D-lactate contents after long-term administration of AA have not

86 been explored, although elevation of urinary D-lactate was observed in a previous

87 study [20].

88 This study aimed to explore whether the progression of tubulointerstitial injury

89 and interstitial renal fibrosis stop and/or reverse after discontinuing 56-day

90 administration of low-dose AA by determining the contents of urinary methylglyoxal

91 and D-lactate. The results of this study may help to identify the safe dose and duration

92 of administration of AA-related products, especially Xixin.

94 Methods

95 Materials and chemicals 


\section{Animal experiments}

All animal protocols were approved by the Animal Care and Use Committee of Taipei Medical University (LAC-2019-0482). Six-week-old female C3H/He mice were obtained from the National Laboratory Animal Breeding and Research Center (Taipei, Taiwan) and randomly allocated to either the normal group $(\mathrm{N})$ or the AA group ( $n=24 /$ group) [20]. Six mice in the same group were placed in per cage. These mice were placed in temperature-controlled $\left(25 \pm 2^{\circ} \mathrm{C}\right)$ and humidity-controlled $(65 \pm$ $5 \%$ ) rooms with a 12:12 light-dark photoperiod, and had access to standardized food pellets (Fwusow Industry Co., LTD., Taichung, Taiwan). The AA group were given ad libitum access to distilled water containing $3 \mu \mathrm{g} / \mathrm{mL}$ AA (Sigma-Aldrich, Inc., MO, USA; AAI:AAII = 63:31;0.5 mg/kg/day) as drinking water for 56 days. After day 56, the AA group mice received normal drinking water [20,21]. The $\mathrm{N}$ group received only normal drinking water. Urine samples were collected over 12 hours on days 28 , 56, 70, and 84 using a metabolic cage (Tokiwa Chemical Industries Co. Ltd., Japan). The mice were humanely sacrificed on day $28,56,70$ or $84(n=6 /$ group $)$. Whole blood samples were collected and the kidneys were excised [20, 21].

\section{Biochemical parameters}


used to determine blood urea nitrogen (BUN). Urinary $N$-acetyl- $\beta$-D-glucosaminidase

116 (NAG) activity, i.e., production of 4-methylumbelliferone (4-MU) from 4glycine buffer ( $\mathrm{pH} 10.4-10.6$ ) and fluorescence was measured at $460 \mathrm{~nm}$ after excitation at $370 \mathrm{~nm}[21]$.

122 protocol $[14,22]$. Briefly, $20 \mu \mathrm{L}$ blood samples were mixed with $10 \mu \mathrm{L}$ of $1 \mathrm{mM}$

123 cimetidine $\cdot \mathrm{HCl}$ (Sigma-Aldrich, Inc., MO, USA) as an internal standard [I.S.] and

$124170 \mu \mathrm{L}$ acetonitrile $(\mathrm{MeCN})$ and centrifuged at $700 \mathrm{~g}$ for $15 \mathrm{~min}$ at $4^{\circ} \mathrm{C}$. The

125 supernatants $(50 \mu \mathrm{L})$ were injected into the UV-HPLC system, which included an L-

1267100 Pump (Hitachi, Tokyo, Japan), L-2200 Intelligent Autosampler (Hitachi),

127 TSKgel ODS-80Ts column $(250 \times 4.6 \mathrm{~mm}$ i.d., $5 \mu \mathrm{m}$; Tosoh Co., Tokyo, Japan $)$ and

128 an 875-UV Intelligent UV/VIS Detector (JASCO International Co., Ltd., Tokyo,

129 Japan). The mobile phase was 36:60 (v/v) MeCN/30 mM sodium lauryl sulfate

130 (aq): $100 \mathrm{mM}$ sodium dihydrogen phosphate $(\mathrm{aq})(\mathrm{pH} 3.0)$ and the flow rate was 0.8

$131 \mathrm{~mL} / \mathrm{min}$. The fractions were monitored at $234 \mathrm{~nm}$.

\section{Histological examinations}


$13424 \mathrm{~h}$ at $4{ }^{\circ} \mathrm{C}$, embedded in paraffin, sectioned at $4-5 \mu \mathrm{m}$ and stained with periodic

135 acid-Schiff (PAS) (Muto Pure Chemicals Co., Ltd., Tokyo, Japan), Masson's

136 trichrome (Muto Pure Chemicals Co., Ltd., Tokyo, Japan), or Picro Sirius Red

137 Staining Kit (CIS-Biotechnology Co., Ltd., Taichung, Taiwan) according to the

138 instruction of manufactures [20, 21]. Sections were observed at a magnification of

$139200 \times$ using a G-330 light microscope (Optima, New Taipei City, Taiwan) and images

140 were captured using a Nikon Coolpix 4500 camera (Nikon, Tokyo, Japan) [20, 21].

\section{Tubulointerstitial histological score}

143 of tubulointerstitial damage in the PAS-stained sections. Ten non-overlapping fields of

144 view were scored for each mouse. The TIHS assesses three major items: the severity

145 of mononuclear cell infiltration into the interstitium ( 0 , absent; 1 , few scattered cells;

146 2, groups of mononuclear cells; and 3, dense widespread infiltrate); the severity of

147 degeneration in the tubular epithelium ( 0 , no degeneration of the tubular epithelium;

1481 , one group or a single degenerated tubule; 2 , several clusters of degenerated tubules;

1493 , moderate degeneration of the tubular epithelium; 4, more severe degeneration of

150 the tubular epithelium; and 5, extremely severe degeneration of the tubular

151 epithelium, with massive necrosis and atrophy); and the severity of interstitial fibrosis 
152 (0, absent; 1 , mild diffuse fibrosis; 2 , moderate fibrosis; and 3, severe fibrosis). The

153 THIS of each mouse was expressed as the sum of the three scores [14, 15, 20, 21, 23].

\section{Semi-quantitative analysis of fibrosis}

155

Interstitial renal fibrosis was assessed in the Masson trichrome and Picro Sirius

Red-stained sections by determining the percentage area positive for aniline blue and

157 red in ten non-overlapping fields of view for each mouse using ImageJ (National

158 Institutes of Health, MD, USA), respectively [20].

\section{Determination of methylglyoxal}

Urinary and serum methylglyoxal were determined by high-performance liquid

161 chromatography with fluorescence detection (FD-HPLC). The FD-HPLC system

162 (Hitachi, Tokyo, Japan) was composed of L-2130 Pump, L-2200 Intelligent

163 Autosampler and L-2480 Fluorescence Detector [13, 20].

165 diamino-2,4-dihydroxypyrimidine sulfate (DDP) for $30 \mathrm{~min}$ at $60^{\circ} \mathrm{C}$, the reactions

166 were stopped with $0.01 \mathrm{M}$ citric acid (pH 6.0) and $20 \mu \mathrm{L}$ samples were loaded onto

167 the ODS column $(250 \times 4.6 \mathrm{~mm}, 5 \mu \mathrm{m}$ particle size; Biosil Chemical Co. Ltd., Taipei,

168 Taiwan) at $33{ }^{\circ} \mathrm{C}$ [24]. The mobile phase was 97:3 (v/v) $0.01 \mathrm{M}$ citric acid buffer (pH

169 6.0) and $\mathrm{MeCN}$ and the flow rate was $0.7 \mathrm{~mL} / \mathrm{min}$. The fractions were monitored at an

170 emission wavelength of $500 \mathrm{~nm}$ and excitation wavelength of $330 \mathrm{~nm}$. Urinary 
171 methylglyoxal content was expressed as the level of methylglyoxal $\times 12$ h-urinary

172 volume [20].

\section{Determination of D-lactate}

\section{Florigenic derivatization of lactate}

$175 \quad$ Urine samples $(20 \mu \mathrm{L})$ were mixed with $10 \mu \mathrm{L}$ of $1 \mathrm{mM}$ propionic acid as an

176 internal standard (I.S.) and $170 \mu \mathrm{L}$ of acetonitrile $(\mathrm{MeCN})$ and centrifuged at $700 \mathrm{~g}$

177 for $10 \mathrm{~min}$ at $4{ }^{\circ} \mathrm{C}$. For florigenic derivatization, $100 \mu \mathrm{L}$ of the supernatant was

178 incubated with $100 \mu \mathrm{L}$ of $8 \mathrm{mM}$ 4-nitro-7-piperazino-2,1,3-benzoxadiazole (NBD-PZ)

179 in $\mathrm{MeCN}$ in $25 \mu \mathrm{L}$ of $280 \mathrm{mM}$ triphenylphosphine (TPP) and $25 \mu \mathrm{L}$ of $280 \mathrm{mM} 2,2$ '-

180 dipyridyl disulfide (DPDS) in MeCN. After 3 h, $250 \mu \mathrm{L}$ of $0.1 \%$ aqueous

181 trifluoroacetic acid (TFA) was added to stop the reaction. To remove excess NBD-PZ,

$182100 \mu \mathrm{L}$ of the solution was passed through a MonoSpin ${ }^{\mathrm{TM}}$ SCX cartridge (GL Science

183 Inc., Tokyo, Japan) and the eluates were collected [20, 25].

\section{Isolation of D,L-lactate and D-lactate}

185 Urinary D-lactate were determined using a column-switching FD-HPLC system

186 by first isolating D,L-lactate and then separating D-lactate. NBD-PZ-fluorogenic

187 derivatized samples $(20 \mu \mathrm{L})$ were loaded into an L-7100 Pump (Hitachi, Tokyo,

188 Japan), L-2200 Intelligent Autosampler (Hitachi, Tokyo, Japan), Biosil ODS column

189 (4.6 $\times 250$ mm, $5 \mu$ m; Biotic Chemical Co., Ltd., Taipei, Taiwan) and L-2485 
210 Waltham, MA, USA). The samples (10 $\mu \mathrm{g}$ protein per lane) were separated on $12 \%$

211 sodium dodecyl sulfate-polyacrylamide gels and electrophoretically transferred onto

212 Immun-Blot PVDF Membranes (Bio-Rad Laboratories, Inc., Hercules, CA, USA)

213 [21]. The antibodies used were anti-glyoxalase 1 antibody (GLO1; GTX105792;

214 GeneTex, Irvine, CA, USA; 1:1000), anti- $\beta$-actin antibody (20536-1-AP; Proteintech,

215 Rosemont, IL, USA; 1:2000) and goat anti-rabbit IgG (H+L) HRP conjugate antibody

216 (SA00001-2; Proteintech, Rosemont, IL, USA ; 1:4000). TOOLSensitive ECL Kit

217 (Tools Biotechnology co. Ltd., New Taipei City, Taiwan) was used detect the bands.

218 The intensity of the bands were measured using Image J (National Institute of Health,

219 Bethesda, Maryland, USA) and the levels of GLO1 were normalized to $\beta$-actin and

220 expressed relative to the $\mathrm{N}$ group.

\section{Statistical analysis}

222 All values were expressed as means \pm standard deviation. The differences

223 between the N and AA groups were assessed using the Student's t-test at each time

224 point. Repeated measures ANOVA was used to compare the mean values of the

225 variables in the same group on Day 56, 70, and 84. P-values less than 0.05 were 
USA) was used to analyze all data.

228

Results

230

\section{Biochemical parameters}

All biochemical parameters (BUN, Scr, and NAG) were significantly elevated in

232 the AA group compared to the $\mathrm{N}$ group on days 28, 56, 70 and 84 (Table 1). There

233 were no significant differences in the BUN and Scr contents of the AA group on day

$23456(24.13 \pm 1.21 \mathrm{mg} / \mathrm{dL}$ and $0.283 \pm 0.041 \mathrm{mg} / \mathrm{dL})$, day $70(25.83 \pm 1.17 \mathrm{mg} / \mathrm{dL}$ and

$2350.317 \pm 0.075 \mathrm{mg} / \mathrm{dL})$, and day $84(24.99 \pm 0.98 \mathrm{mg} / \mathrm{dL}$ and $0.300 \pm 0.063 \mathrm{mg} / \mathrm{dL})$. In

236 the AA group, urinary NAG activity was higher on day $56(2.079 \pm 0.089 \mathrm{U} / \mathrm{L})$ and

237 day $70(2.297 \pm 0.266 \mathrm{U} / \mathrm{L})$ than on day $84(1.895 \pm 0.110 \mathrm{U} / \mathrm{L})$.

\section{Histological examination}

239 PAS stained kidney sections (Fig. 1) indicated exposure to AA induced

240 interstitial renal fibrosis, with the most severe fibrosis observed on day 70 (Fig. 1f).

241 The PAS staining sections revealed severe renal damage in the AA group, including

242 cellular infiltration, epithelial damage and interstitial renal fibrosis. There was a

243 slightly cell infiltration and tubular atrophy on day 28 , but no significant difference in

244 TIHS between AA $(0.8 \pm 0.6)$ and $\mathrm{N}(0.2 \pm 0.4)$ groups (Fig. 1a,1b,1f). The AA group 
245 had significantly higher TIHS than the $\mathrm{N}$ group on day 56 (AA: $3.6 \pm 0.7 v s$. N: $0.2 \pm$ $2460.4 ; p<0.01$ ), day 70 (AA: $6.3 \pm 0.8 v s . \mathrm{N}: 0.3 \pm 0.7 ; p<0.01$ ), and day 84 (AA: 4.6

$247 \pm 0.5 v s . \mathrm{N}: 0.3 \pm 0.7 ; p<0.01$ ) (Fig. 1f). The TIHS of the AA group increased 248 between day 28 and day 70 and then reduced by day 84 (Fig. 1f).

249 Masson's trichrome staining was performed to semi-quantitatively analyze

250 collagen deposition (Fig. 2). There was no significant difference in collagen

251 deposition between AA $(3.6 \pm 0.2 \%)$ and N $(3.5 \pm 0.4 \%)$ groups on day 28 (Fig.

252 2a,b,i). Collagen deposition was significantly higher in the AA group than the $\mathrm{N}$ group

253 on day 56 (AA: $14.1 \pm 2.3 \%$ vs. N: $3.6 \pm 0.5 \% ; p<0.01$ ), day 70 (AA: $28.5 \pm 3.5 \%$

254 vs. N: $3.8 \pm 0.4 \% ; p<0.01$ ), and day 84 (AA: $20.6 \pm 1.6 \%$ vs. N: $4.0 \pm 1.0 \% ; p<$

255 0.01) (Fig. 2i). In the AA group, higher levels of collagen deposition were detected on

256 day 70 (Fig. 2f) than on days 56 (Fig. 2d) and 84 (Fig. 2h). Moreover, collagen

257 deposition was also assessed by Picro Sirius Red staining (Fig. 3), and the findings

258 were similar to Masson's trichrome staining. There was no significant difference in

259 collagen deposition between AA $(5.86 \pm 1.38 \%)$ and $\mathrm{N}(5.72 \pm 1.20 \%)$ groups on day

26028 (Fig. 3a,b,i). The collagen deposition was significantly higher in the AA group than

261 the $\mathrm{N}$ from day 56 (AA: $11.3 \pm 1.2 \%$ vs. $\mathrm{N}: 6.5 \pm 1.3 \% ; p<0.05)$ to day 84 (AA: 15.7

$262 \pm 1.5 \%$ vs. N: $6.4 \pm 1.5 \% ; p<0.05$ ) (Fig. 3i). The highest levels of collagen

263 deposition in the AA group on day 70 (Fig. 3f), and the collagen deposition 
(Fig. 3i).

266

\section{Methylglyoxal in serum and urine}

The AA group had a higher serum methylglyoxal level than the $\mathrm{N}$ group on day 56 (AA: $429.4 \pm 48.3 \mu \mathrm{g} / \mathrm{L} v s . \mathrm{N}: 311.9 \pm 29.2 \mu \mathrm{g} / \mathrm{L} ; p<0.05$ ), day 70 (AA: $878.3 \pm$

$171.8 \mu \mathrm{g} / \mathrm{L} v s . \mathrm{N}: 373.1 \pm 52.9 \mu \mathrm{g} / \mathrm{L} ; p<0.01$ ), and day 84 (AA: $600.2 \pm 99.9 \mu \mathrm{g} / \mathrm{L} v s$.

\section{$\mathrm{N}: 386.6 \pm 61.5 \mu \mathrm{g} / \mathrm{L} ; p<0.05$ ) (Fig. 4d). In the AA group, the serum methylglyoxal}

level content significantly increased between day 56 and day 70 and declined between day 70 and day 84 (Fig. 4d).

The AA group had higher urine methylglyoxal contents than the $\mathrm{N}$ group on day 56 (AA: $3.522 \pm 1.061 \mu \mathrm{g} v s . \mathrm{N}: 1.408 \pm 0.135 \mu \mathrm{g} ; p<0.05)$ and day 84 (AA: $2.390 \pm$ $0.130 \mu \mathrm{g} v s . \mathrm{N}: 1.630 \pm 0.081 \mu \mathrm{g} ; p<0.05)($ Fig. $4 \mathrm{~h})$. There was no significant difference in the urinary methylglyoxal contents of the AA $(1.583 \pm 0.437 \mu \mathrm{g})$ and $\mathrm{N}$ groups $(1.386 \pm 0.255 \mu \mathrm{g})$ on day 70 (Fig. $4 \mathrm{~h})$. The urinary methylglyoxal content of the AA group significantly decreased between day 56 and 70 and significantly increased between day 70 and 84 (Fig. 4h).

\section{Urinary D,L-lactate and D-lactate contents}

The AA group had significantly higher urinary D,L-lactate contents than the $\mathrm{N}$ group on day 56 (AA: $994.1 \pm 161.0 \mu \mathrm{g} v s . \mathrm{N}: 151.9 \pm 71.1 \mu \mathrm{g} ; p<0.001)$, day 70 
283

284

285

286

287

288

289

290

291

292

293

294

295

296

297

298

299

300

(AA: $1598.9 \pm 396.0 \mu \mathrm{g} v s . \mathrm{N}: 125.8 \pm 57.4 \mu \mathrm{g} ; p<0.001$ ), and day 84 (AA: $1447.0 \pm$

$531.4 \mu \mathrm{g} v s . \mathrm{N}: 141.5 \pm 48.6 \mu \mathrm{g} ; p<0.001$ ) (Fig. $5 \mathrm{~g}$ ). There was no significant change in the urinary D,L-lactate content of the $\mathrm{N}$ group from day 56 to 84 . However, the urinary D/L-lactate content of the AA group significantly increased between day 56 and day $70(p<0.05)$ and remained high at day 84 (Fig. $5 \mathrm{~g})$.

The urinary D-lactate contents of the AA group were significantly higher than those of the $\mathrm{N}$ group on day 56 (AA: $82.10 \pm 18.80 \mu \mathrm{g} v s . \mathrm{N}: 5.88 \pm 3.40 \mu \mathrm{g} ; p<$ 0.001 ), day 70 (AA: $201.09 \pm 90.82 \mu \mathrm{g} v s . \mathrm{N}: 5.78 \pm 2.77 \mu \mathrm{g} ; p<0.001$ ), and day 84 (AA: $193.28 \pm 61.32 \mu \mathrm{g} v s . \mathrm{N}: 7.15 \pm 3.28 \mu \mathrm{g} ; p<0.001$ ) (Fig. $5 \mathrm{~h}$ ). The urinary Dlactate amount of the $\mathrm{N}$ group did not significantly change between day 56 and 84 .

However, the urinary D-lactate amount of the AA group significantly increased between day 56 to $70(p<0.05)$ and remained high at day 84 (Fig. 5 h).

\section{Expression of GLO1}

The relative levels of GLO1 in the kidney of the AA group on day 56 (180.9 \pm $12.8 \%)$, day $70(150.8 \pm 14.9 \%)$, and day $84(167.2 \pm 13.3 \%)$ were significantly higher than the $\mathrm{N}$ group $(100.0 \pm 9.0 \% ; p<0.05)$ (Fig. $6 \mathrm{~b})$.

\section{Discussion}



human study by Vanhaelen et al. (about $130 \mathrm{mg}$ ) [26]. The concentration of AA in

313 still available in markets [28]. Thus, it is necessary to determine the AA

314 concentrations of Xixin crude herbs and concentrated granule samples. 
320 AA (10 mg/kg/day) for five weeks; however, the changes after withdrawal of AA

321 were not observed [30, 31]. In the current study, slight elevation of biochemical

322 parameters were observed but rare fibrosis by day 28, compared to those on day 56

323 and 70. Moreover, while another acute mouse model used a much higher dose (10

$324 \mathrm{mg} / \mathrm{kg} /$ day $)$ than the current study $(0.5 \mathrm{mg} / \mathrm{kg} / \mathrm{day})$, tubular necrosis — but rarely

325 interstitial renal fibrosis - were observed after intravenous administration of AA for

326 five days $[14,16]$. Consequently, administration of a low cumulative dose (i.e., a low

327 dose for a short period) of Xixin and monitoring NAG and renal function are essential

328 to avoid exacerbation of tubulointerstitial injury.

329 Methylglyoxal and D-lactate are regarded as biomarkers for nephropathy and

330 diabetes, thus most previous studies focused on how methylglyoxal is induced

331 endogenously, how methylglyoxal reacts with proteins and nucleic acids to produce

332 AGEs [27], and testing drugs that may lower methylglyoxal contents. Therefore,

333 urinary excretion of methylglyoxal and D-lactate are considered to reflect excessive

334 production of methylglyoxal due to renal injury and inflammation. Based on the

335 biochemical parameters and renal biopsies in this study, cell infiltration and renal

336 injury such as tubule degeneration and moderate fibrosis truly existed in the injured

337 kidney after administration of AA for 56 days. In a previous study, inflammation

338 markers, such as F4/80 and tumor necrosis factor- $\alpha$ (TNF- $\alpha)$, were induced in the 
339 damaged kidney after long-term administration of AA [21]. On the other hand,

340 methylglyoxal is produced after injury or inflammation and is also a pro-

341 inflammatory factor. Methylglyoxal promotes inflammation via upregulating the

342 nuclear factor-kappa B (NF-кB) signaling pathway [28]. Furthermore, AGEs,

343 methylglyoxal-derivatized adducts, react with receptors for advanced glycation end

344 products (RAGE) to further evoke inflammation [32].

345 Another essential finding of this study was that the level of serum methylglyoxal

346 peaked on day 70, while the levels of MG excreted in urine were lower at this time

347 point than at day 70.These findings indirectly reflects accumulation of methylglyoxal

348 in the injured kidney. In agreement with these observations, severe renal injury and

349 interstitial fibrosis were detected at day 70. As previously described, accumulation of

350 methylglyoxal is harmful and aggravates fibrosis in a variety of pathologies [33-35].

351 The potential mechanisms include activation of transient receptor potential ankyrin 1

352 (TRPA1) [35], promotion of the cell cycle [35], differentiation of fibroblasts [33-36],

353 induction of the epithelial-mesenchymal transition (EMT) via the TGF- $\beta$ /Snail axis

354 [34], and inhibition of the binding step of collagen phagocytosis [37]. Moreover,

355 Kottmann et al. proposed that lactic acid may activate TGF- $\beta$ and lead to

356 accumulation of hypoxia-inducible factor 1- $\alpha$ (HIF-1 $\alpha)$ - which promotes 
357

myofibroblast differentiation—in idiopathic pulmonary fibrosis [38]. However, the effects of methylglyoxal on interstitial renal fibrosis are still unknown.

$$
\text { Previous studies only assessed urinary methylglyoxal and D-lactate under injury }
$$

conditions, such as oxidative stress [39], inflammation [40], necrosis [14] or fibrosis

[20]). However, we found that urinary methylglyoxal and D-lactate remained elevated at 28 days after AA withdrawal (day 84), when the severity of fibrosis and tubulointerstitial injury had reduced to mild. These changes in urinary methylglyoxal and D-lactate may be related to renal repair and detoxification. Generally, injured tissues use glycolysis to produce energy for repair [41], thus methylglyoxal might be released. Lan et al. detected increased levels of byproducts of glycolysis (lactate and pyruvate) in the kidney during reperfusion after acute ischemia-reperfusion injury [42]. Thus, methylglyoxal may be excreted into urine and metabolized into D-lactate by GLO1 as a mechanism of detoxification [18]. Indeed, most studies did not assess the pathological alterations after long-term withdrawal of AA. However, our assessment of the changes up to 28 days after withdrawal of AA (day 84) indicate repair is a time-consuming process. The association between repair and methylglyoxal warrants more detailed investigation in further research. Moreover-as a limitation of this study—although AA-DNA adducts are a biomarker for AA exposure and urothelial cell carcinoma [43], AA-DNA adducts were not determined in this study. In 
376 the future, AA-DNA adducts should be detected to evaluate the severity of

377 tubulointerstitial injury.

378

379 Conclusion

380

Methylglyoxal is produced and exacerbates kidney injury (inflammation and

381 fibrosis), and excretion and metabolism of methylglyoxal may represent a strategy of

382 detoxification after injury. After withdrawal of AA, tubulointerstitial injury became

383 mild, due to the low cumulative dose of AA. Thus, AA-containing herbs such as Xixin

384 should be used at low doses for short durations (i.e., less than one month) and the

385 renal function of the patients should be monitored.

386

387

\section{List of abbreviations}

388 AA: aristolochic acid; AAI: aristolochic acid I; AA II: aristolochic acid II; AAN:

389 aristolochic acid nephropathy; AGEs: advanced glycation end products; BUN: blood

390 urea nitrogen; DDP: 2,20-dipyridyl disulfide; DPDS: 6-diamino-2,4-

391 dihydroxypyrimidine sulfate; EMT: epithelial-mesenchymal transition;

392 FD-HPLC: high-performance liquid chromatography and fluorescence detection;

393 GLO1: glyoxalase 1; HIF-1 $\alpha$ : hypoxia-inducible factor 1- $\alpha$; MeCN: acetonitrile; 4-

394 MU: 4-methylumbelliferone; NAG: $N$-acetyl- $\beta$-D-glucosaminidase; NBD-PZ: 4-nitro- 
395 7-piperazino-2,1,3-benzoxadiazole; NF-кB: nuclear factor-kappa B; OAT: organic

396 anion transporter; PAS: periodic acid-Schiff; PTEC: proximal tubular epithelial cells;

397 RAGE: receptors for advanced glycation end products; Scr: serum creatinine; SSAO:

398 semicarbazide-sensitive amine oxidase; TFA: trifluoroacetic acid; THIS:

399 tubulointerstitial histological score; TNF- $\alpha$ : tumor necrosis factor- $\alpha$; TPP:

400 triphenylphosphine; TRPA1: transient receptor potential ankyrin 1.

401

402 Declarations

403 Ethics approval and consent to participate

404 All animal protocols were approved by the Animal Care and Use Committee of Taipei

405 Medical University (LAC-2019-0482).

406 Consent for publication

407 Not applicable.

408 Availability of data and materials

409 Not applicable.

410 Competing interests

411 The authors declare that they have no competing interests.

$412 \quad$ Funding 
413 We are grateful to the financial support from the Ministry of Science and Technology,

414 Taiwan, R.O.C. (NSC 97-2320-B-038-007-MY3).

415 Authors' contributions

416 Study design and conception of animal experiment: Shih-Ming Chen and Chia-En

417 Lin; data collection and data analysis: Chia-En Lin, Po-Yeh Lin, Wen-Chi Yang, Tzu-

418 Yao Lin, and Chien-Ming Chen; animal experiment: Chia-En Lin, Po-Yeh Lin, Wen-

419 Chi Yang, Yu-Shen Huang, Tzu-Yao Lin; data interpretation: Jen-Ai Lee and Chia-En

420 Lin; manuscript drafting: Chia-En Lin; semi-quantitative analysis for collagen

421 deposition: Hung-Shing Chen; critical revising the manuscript: Shih-Ming Chen and

422 Jen-Ai Lee. All authors reviewed and approved the manuscript.

423 Acknowledgements

424 We appreciate it very much that Prof Shiro Ueda provided recommendation to

425 our animal experiments and metabolic cages for urine collection. The graphical

426 abstract was created with $\underline{B i o R e n d e r . c o m}$. We are grateful to the financial support

427 from the Ministry of Science and Technology (NSC 97-2320-B-038-007-MY3). We

428 acknowledge UNIVERSAL LINK CO., LTD. who provided professional writing

429 services or materials.

430 Footnotes

431 Table 1. Biochemical parameters of the normal (N) and aristolochic acid (AA) group 
432 BUN: blood urea nitrogen; Scr: serum creatinine; NAG: $N$-acetyl- $\beta-D-$

433 glucosaminidase; * $p<0.05$ vs. N group at the same time point, Student's t-test; \# $p<$ $4340.05 v s$. AA group on day $56 ;{ }^{\dagger} p<0.05 v s$. AA group on day 70 , repeated measures 435 ANOVA

436 Fig. 1 Periodic acid-Schiff (PAS) stained kidney sections and tubular interstitial 437 histological scores (TIHS)

438 (a-i) Representative images of PAS-stained kidney sections from the $\mathrm{N}$ group on day $43928(\mathrm{a}), 56(\mathrm{c}), 70$ (e), and 84 (g) and AA group on days 28 (b), 56 (d), 70 (f), and 84

440 (h). The arrow indicates interstitial renal fibrosis. The stars indicate cellular

441 infiltration. (i) Tubular interstitial histological scores (THIS). N group, normal group;

442 AA group, aristolochic acid group. ${ }^{* *} p<0.01 v s . \mathrm{N}$ group at the same time point,

443 Student's $t$-test; \# $p<0.05$ vs. AA group on day $56 . \dagger p<0.05 v s$. AA group on day

44470 , repeated measures ANOVA.

445 Fig. 2 Masson's trichrome stained kidney sections and semi-quantitative analysis of

446 collagen deposition

447 The fraction of interstitial fibrosis was assessed as the percentage of aniline blue-

448 stained area. (a-i) Representative images of Masson's trichrome-stained kidney

449 sections from the $\mathrm{N}$ group on day 28 (a), 56 (c), day 70 (e), and day 84 (g) and AA

450 group on day 28 (b), 56 (d), day 70 (f), and day 84 (h). The arrow indicates collagen 
451 deposition. (i) Semiquantitative analysis of collagen deposition. N group, normal

452 group; AA group, aristolochic acid group. ** $p<0.01$ vs. $\mathrm{N}$ group at the same time

453 point, Student's $t$-test; \# $p<0.05$ vs. AA group on day 56. $\dagger p<0.05 v s$. AA group on

454 day 70, repeated measures ANOVA.

455 Fig. 3 Picro Sirius Red stained kidney sections and semi-quantitative analysis of

456 collagen deposition

457 The fraction of interstitial fibrosis was assessed as the percentage of red-stained area.

458 (a-f) Representative images of Picro Sirius Red-stained kidney sections from the N

459 group on day 28 (a), 56 (c), day 70 (e), and day 84 (g) and AA group on day 28(b), 56

460 (d), day 70 (f), and day 84 (h). The arrow indicates collagen deposition. (i)

461 Semiquantitative analysis of collagen deposition. N group, normal group; AA group,

462 aristolochic acid group. $* p<0.05, * * p<0.01 v s . \mathrm{N}$ group at the same time point,

463 Student's $t$-test; \# $p<0.05$ vs. AA group on day $56 . \dagger p<0.05 v s$. AA group on day

46470 , repeated measures ANOVA.

465 Fig. 4 Chromatograms of analysis and contents of methylglyoxal in serum and urine

466 HPLC chromatograms for serum methylglyoxal level on day 56 (a), day 70 (b), and

467 day 84 (c). The corresponding peaks of methylglyoxal derivative was labeled in

468 HPLC chromatograms. (d) Serum methylglyoxal content. The white bars indicate the

$469 \mathrm{~N}$ group and the black bars indicate the AA group. N group, normal group; AA group, 
470 aristolochic acid group. HPLC chromatograms for urinary methylglyoxal on day 56

471 (e), day 70 (f), and day 84 (g). The corresponding peaks of methylglyoxal derivative

472 was labeled in HPLC chromatograms. (h) Urinary methylglyoxal content, calculated

473 as methylglyoxal level $\times 12$-h urinary volume. N group, normal group; AA group,

474 aristolochic acid group. $* p<0.05$ and $* * p<0.01 v s . \mathrm{N}$ group at the same time point,

475 Student's $t$-test; \# $p<0.05 v s$. AA group on day 56. $\dagger p<0.05 v s$. AA group on day

476 70, repeated measures ANOVA.

477 Fig. 5 Chromatographs of analysis and contents of lactate (D,L-lactate and D-lactate)

478 in urine

479 (a, c, e) HPLC chromatographs for the D,L-lactate on day 56 (a), day 70 (c), and day

48084 (e). (b, d, f) HPLC chromatographs for the D-lactate on day 56 (a), day 70 (b), and

481 day 84 (c). The corresponding peaks of D,L-lactate and D-lactate derivative and

482 internal standard (I.S.) was labeled in HPLC chromatograms. (g, h) Quantification of

483 D,L-lactate (g) and D-lactate (h) amount. Urinary D,L-lactate amount, calculated as

484 D,L-lactate level $\times 12$-h urinary volume; urinary D-lactate amount, calculated as D-

485 lactate level $\times 12$-h urinary volume. $\mathrm{N}$ group, normal group; AA group, aristolochic

486 acid group; I.S., internal standard. ${ }^{* * *} p<0.001 v s . \mathrm{N}$ group at the same time point,

487 Student's $t$-test; \# $p<0.05$ vs. the AA group on day 56, repeated measures ANOVA.

488 Fig. 6 Western blot analysis and relative levels of glyoxalase 1(GLO1) in the kidney 
(a) Representative western blot of GLO-1; $\beta$-actin was used as an internal control. (b)

491 Semi-quantitative analysis of GLO1 expression. AA56, AA group on day 56; AA70,

492 AA group on day 70; AA84, AA group on day 84; N group, normal group; AA group,

493 aristolochic acid group. ${ }^{*} p<0.05$ vs. $\mathrm{N}$ group, ANOVA.

494

495 References

496 1. Zhang C, Wang X, Shang M, Yu J, Xu Y, Li Z, et al. Simultaneous determination of

497 five aristolochic acids and two aristololactams in Aristolochia plants by high-

498 performance liquid chromatography. Biomed Chromatogr. 2006;20(4):309-18.

499 2. Vanherweghem JL, Depierreux M, Tielemans C, Abramowicz D, Dratwa M, Jadoul

$500 \mathrm{M}$, et al. Rapidly progressive interstitial renal fibrosis in young women: association

501 with slimming regimen including Chinese herbs. Lancet. 1993;341(8842):387-91.

502 3. Kamei T, Kondoh T, Nagura S, Toriumi Y, Kumano H, Tomioka H. Improvement of

503 C-reactive protein levels and body temperature of an elderly patient infected with

504 Pseudomonas aeruginosa on treatment with Mao-bushi-saishin-to. J Altern

505 Complement Med. 2000;6(3):235-9.

506 4. Takagi Y, Higashi N, Kawai S, Maeda A, Ueba N. Effects of traditional oriental

507 medicine on influenza virus infection:: Activating effect of Mao-Bushi-Saishin-To 
508 (MBST, Ma-Huang-Fu-Zi-Xin-Tang) on IgG antibody producibility in aged mice.

509 International Congress Series. 2004;1263:670-3.

510 5. Oh J, Hwang IH, Kim DC, Kang SC, Jang TS, Lee SH, et al. Anti-listerial

511 compounds from Asari Radix. Arch Pharm Res. 2010;33(9):1339-45.

512 6. Hashimoto K, Yanagisawa T, Okui Y, Ikeya Y, Maruno M, Fujita T. Studies on anti-

513 allergic components in the roots of Asiasarum sieboldi. Planta Med.

$514 \quad 1994 ; 60(2): 124-7$

515 7. Yu HH, Seo SJ, Hur JM, Lee HS, Lee YE, You YO. Asarum sieboldii extracts

516 attenuate growth, acid production, adhesion, and water-insoluble glucan synthesis

517 of Streptococcus mutans. J Med Food. 2006;9(4):505-9.

518 8. Kim SJ, Gao Zhang C, Taek Lim J. Mechanism of anti-nociceptive effects of

519 Asarum sieboldii Miq. radix: potential role of bradykinin, histamine and opioid

520 receptor-mediated pathways. J Ethnopharmacol. 2003;88(1):5-9.

521 9. Ren M, Tang Q, Chen F, Xing X, Huang Y, Tan X. <i>Mahuang Fuzi Xixin</i>

522 Decoction Attenuates Th1 and Th2 Responses in the Treatment of Ovalbumin-

523 Induced Allergic Inflammation in a Rat Model of Allergic Rhinitis. Journal of

524 Immunology Research. 2017;2017:8254324.

525 10.Zhang W, Zhang J, Zhang M, Nie L. Protective effect of Asarum extract in rats

526 with adjuvant arthritis. Exp Ther Med. 2014;8(5):1638-42. 
527 11.Beisswenger PJ, Drummond KS, Nelson RG, Howell SK, Szwergold BS, Mauer

528 M. Susceptibility to diabetic nephropathy is related to dicarbonyl and oxidative

$529 \quad$ stress. Diabetes. 2005;54(11):3274-81.

530 12.Li YC, Shih YM, Lee JA. Gentamicin caused renal injury deeply related to

531 methylglyoxal and N(varepsilon)-(carboxyethyl)lysine (CEL). Toxicol Lett.

$532 \quad 2013 ; 219(1): 85-92$.

533 13.Huang YS, Li YC, Tsai PY, Lin CE, Chen CM, Chen SM, et al. Accumulation of

534 methylglyoxal and d-lactate in $\mathrm{Pb}$-induced nephrotoxicity in rats. Biomed

535 Chromatogr. 2017;31(5).

536 14.Huang TC, Chen SM, Li YC, Lee JA. Urinary d-lactate levels reflect renal function

537 in aristolochic acid-induced nephropathy in mice. Biomed Chromatogr. 2013;27(9):

$538 \quad 1100-6$.

539 15.Huang TC, Chen SM, Li YC, Lee JA. Increased renal semicarbazide-sensitive

540 amine oxidase activity and methylglyoxal levels in aristolochic acid-induced

541 nephrotoxicity. Life Sci. 2014;114(1):4-11.

542 16.Li YC, Tsai SH, Chen SM, Chang YM, Huang TC, Huang YP, et al. Aristolochic

543 acid-induced accumulation of methylglyoxal and Nepsilon-(carboxymethyl)lysine:

544 an important and novel pathway in the pathogenic mechanism for aristolochic acid

545 nephropathy. Biochem Biophys Res Commun. 2012;423(4):832-7. 
546 17.Thornalley PJ. Protein and nucleotide damage by glyoxal and methylglyoxal in

547 physiological systems--role in ageing and disease. Drug Metabol Drug Interact.

$548 \quad 2008 ; 23(1-2): 125-50$.

549 18.Thornalley PJ. Glyoxalase I--structure, function and a critical role in the enzymatic

550 defence against glycation. Biochem Soc Trans. 2003;31(Pt 6):1343-8.

551 19.Vander Jagt DL. Glyoxalase II: molecular characteristics, kinetics and mechanism.

$552 \quad$ Biochem Soc Trans. 1993;21(2):522-7.

553 20.Chen SM, Lin CE, Chen HH, Cheng YF, Cheng HW, Imai K. Effect of

554 prednisolone on glyoxalase 1 in an inbred mouse model of aristolochic acid

555 nephropathy using a proteomics method with fluorogenic derivatization-liquid

556 chromatography-tandem mass spectrometry. PLoS One. 2020;15(1):e0227838.

557 21.Lin CE, Chang WS, Lee JA, Chang TY, Huang YS, Hirasaki Y, et al. Proteomics

558 analysis of altered proteins in kidney of mice with aristolochic acid nephropathy

559 using the fluorogenic derivatization-liquid chromatography-tandem mass

560 spectrometry method. Biomed Chromatogr. 2018;32(3).

561 22.Marsilio R, Dall'Amico R, Giordano G, Murer L, Montini G, Ros M, et al. Rapid

562 determination of creatinine in serum and urine by ion-pair high-performance liquid

563 chromatography. Int J Clin Lab Res. 1999;29(3):103-9.

564 23.Sato N, Takahashi D, Chen SM, Tsuchiya R, Mukoyama T, Yamagata S, et al. 
565 Acute nephrotoxicity of aristolochic acids in mice. J Pharm Pharmacol.

$566 \quad 2004 ; 56(2): 221-9$.

567 24.Espinosa-Mansilla A, Duran-Meras I, Canada FC, Marquez MP. High-performance

568 liquid chromatographic determination of glyoxal and methylglyoxal in urine by

569 prederivatization to lumazinic rings using in serial fast scan fluorimetric and diode

$570 \quad$ array detectors. Anal Biochem. 2007;371(1):82-91.

571 25.Lee J-A, Tsai Y-C, Chen H-Y, Wang C-C, Chen S-M, Fukushima T, et al.

572 Fluorimetric determination of d-lactate in urine of normal and diabetic rats by

573 column-switching high-performance liquid chromatography. Analytica Chimica

$574 \quad$ Acta. 2005;534(2):185-91.

575 26.Vanhaelen M, Vanhaelen-Fastre R, But P, Vanherweghem JL. Identification of

576 aristolochic acid in Chinese herbs. Lancet. 1994;343(8890):174.

577 27.Jong TT, Lee MR, Hsiao SS, Hsai JL, Wu TS, Chiang ST, et al. Analysis of

578 aristolochic acid in nine sources of Xixin, a traditional Chinese medicine, by liquid

579 chromatography/atmospheric pressure chemical ionization/tandem mass

580 spectrometry. J Pharm Biomed Anal. 2003;33(4):831-7.

581 28.Hsu Y-H, Lo C-F, Liu F-S, Lin J-H. Analysis of Aristolochic Acid in Asarum

582 (Xixin) and Its Preparations by Liquid Chromatography/Tandem Mass

583 Spectrometry. Journal of Food and Drug Analysis. 2009;17:274-81. 
585 Chronic aristolochic acid toxicity in rabbits: a model of Chinese herbs

586 nephropathy? Kidney Int. 2001;59(6):2164-73.

587 30.Debelle FD, Nortier JL, De Prez EG, Garbar CH, Vienne AR, Salmon IJ, et al.

588 Aristolochic acids induce chronic renal failure with interstitial fibrosis in salt-

589 depleted rats. J Am Soc Nephrol. 2002;13(2):431-6.

590 31.Lebeau C, Debelle FD, Arlt VM, Pozdzik A, De Prez EG, Phillips DH, et al. Early

591 proximal tubule injury in experimental aristolochic acid nephropathy: functional

592 and histological studies. Nephrology Dialysis Transplantation. 2005;20(11):2321-

59332.

594 32.Rabbani N, Thornalley PJ. Glyoxalase Centennial conference: introduction, history

595 of research on the glyoxalase system and future prospects. Biochem Soc Trans.

$596 \quad 2014 ; 42(2): 413-8$.

597 33.Retamal IN, Hernandez R, Melo F, Zapata P, Martinez C, Martinez J, et al.

598 Glycated Collagen Stimulates Differentiation of Gingival Myofibroblasts. J

$599 \quad$ Periodontol. 2017;88(9):926-35.

600 34.Hirahara I, Ishibashi Y, Kaname S, Kusano E, Fujita T. Methylglyoxal induces

601 peritoneal thickening by mesenchymal-like mesothelial cells in rats. Nephrol Dial

602 Transplant. 2009;24(2):437-47. 
604 of methylglyoxal on human cardiac fibroblast: roles of transient receptor potential

605 ankyrin 1 (TRPA1) channels. Am J Physiol Heart Circ Physiol.

606 2014;307(9):H1339-52.

607 36.Yuen A, Laschinger C, Talior I, Lee W, Chan M, Birek J, et al. Methylglyoxal-

608 modified collagen promotes myofibroblast differentiation. Matrix Biol.

$609 \quad 2010 ; 29(6): 537-48$.

610 37.Chong SA, Lee W, Arora PD, Laschinger C, Young EW, Simmons CA, et al.

611 Methylglyoxal inhibits the binding step of collagen phagocytosis. J Biol Chem.

$612 \quad 2007 ; 282(11): 8510-20$.

613 38.Kottmann RM, Kulkarni AA, Smolnycki KA, Lyda E, Dahanayake T, Salibi R, et

614 al. Lactic acid is elevated in idiopathic pulmonary fibrosis and induces

615 myofibroblast differentiation via $\mathrm{pH}$-dependent activation of transforming growth

616 factor-beta. Am J Respir Crit Care Med. 2012;186(8):740-51.

617 39.Chen SM, Chen TH, Chang HT, Lin TY, Lin CY, Tsai PY, et al. Methylglyoxal and

618 D-lactate in cisplatin-induced acute kidney injury: Investigation of the potential

619 mechanism via fluorogenic derivatization liquid chromatography-tandem mass

620 spectrometry (FD-LC-MS/MS) proteomic analysis. PLoS One.

$621 \quad 2020 ; 15(7): \mathrm{e} 0235849$. 
622 40.Lin P-Y, Chen S-M, Hsieh C-L, Lin C-Y, Huang Y-S, Hamase K, et al.

623 Determination of temporal changes in serum and urinary lactate and 3-

624 hydroxybutyrate enantiomers in mice with nephrotoxic serum nephritis by multi-

625 dimensional HPLC. Journal of pharmaceutical and biomedical analysis.

$626 \quad 2020 ; 188: 113367$.

627 41.Im MJ, Hoopes JE. Energy metabolism in healing skin wounds. J Surg Res.

$628 \quad 1970 ; 10(10): 459-64$.

629 42.Lan R, Geng H, Singha PK, Saikumar P, Bottinger EP, Weinberg JM, et al.

630 Mitochondrial Pathology and Glycolytic Shift during Proximal Tubule Atrophy

631 after Ischemic AKI. J Am Soc Nephrol. 2016;27(11):3356-67.

632 43.Stiborová M, Arlt VM, Schmeiser HH. DNA Adducts Formed by Aristolochic Acid

633 Are Unique Biomarkers of Exposure and Explain the Initiation Phase of Upper

634 Urothelial Cancer. International journal of molecular sciences. 2017;18(10):2144. 


\section{Figures}
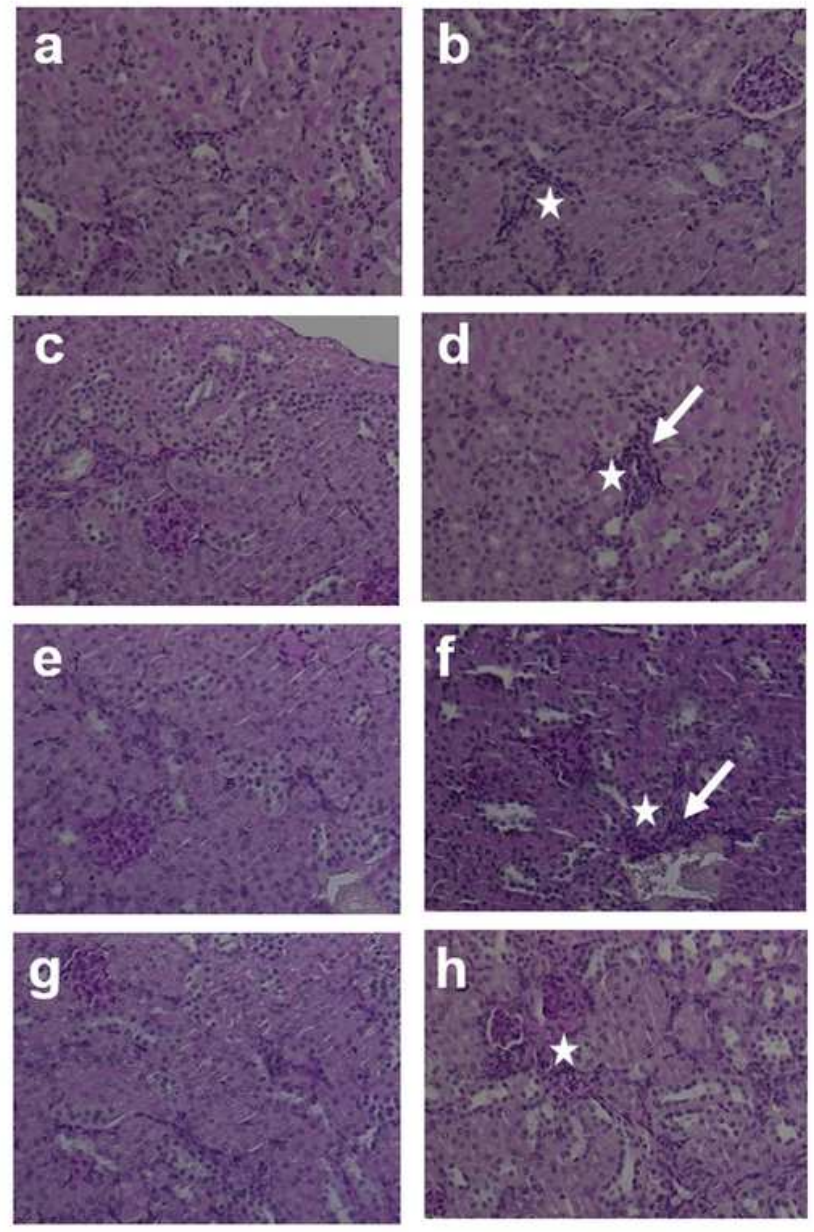
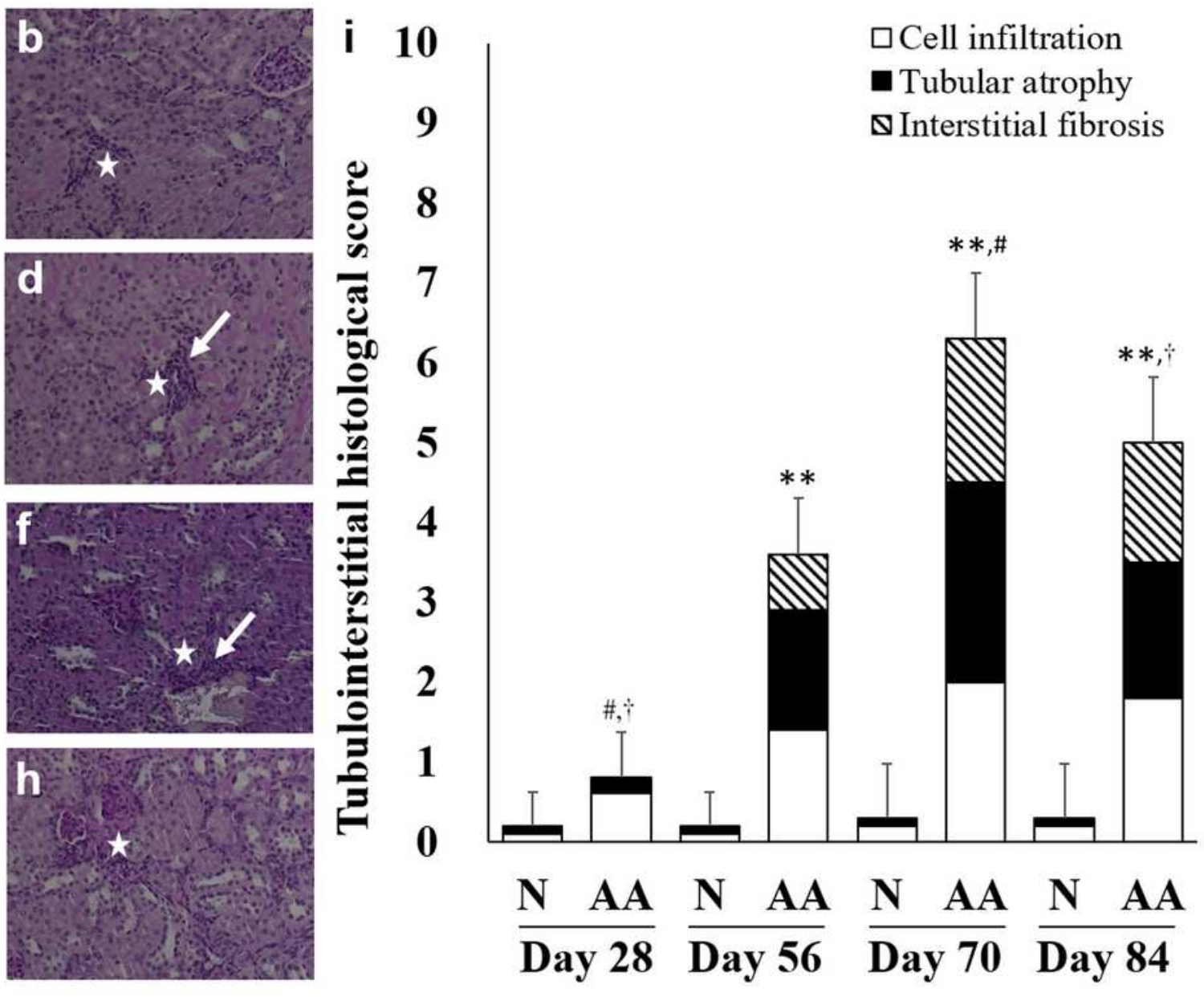

Figure 1

Periodic acid Schiff (PAS) stained kidney sections and tubular interstitial histological scores (TIHS) (a-i ) Representative images of PAS stained kidney section $s$ from the $\mathrm{N}$ group on day 28 (a), 56 (c), 70 (e), and 84 (g) and AA group on days 28 (b), 56 (d), 70 (f), and 84 (h). The arrow indicates interstitial renal fibrosis. The stars in dicate cellular infiltration. (i) T ubular interstitial histological scores (THIS). N group , normal group; AA group, aristolochic acid group. ${ }^{*} p<0.01$ vs. $N$ group at the same time point, Student's $t$ test; $\# p<0.05$ vs. AA group on day 56. $p<0.05$ vs. AA group on day 70 , repeated measures ANOVA. 

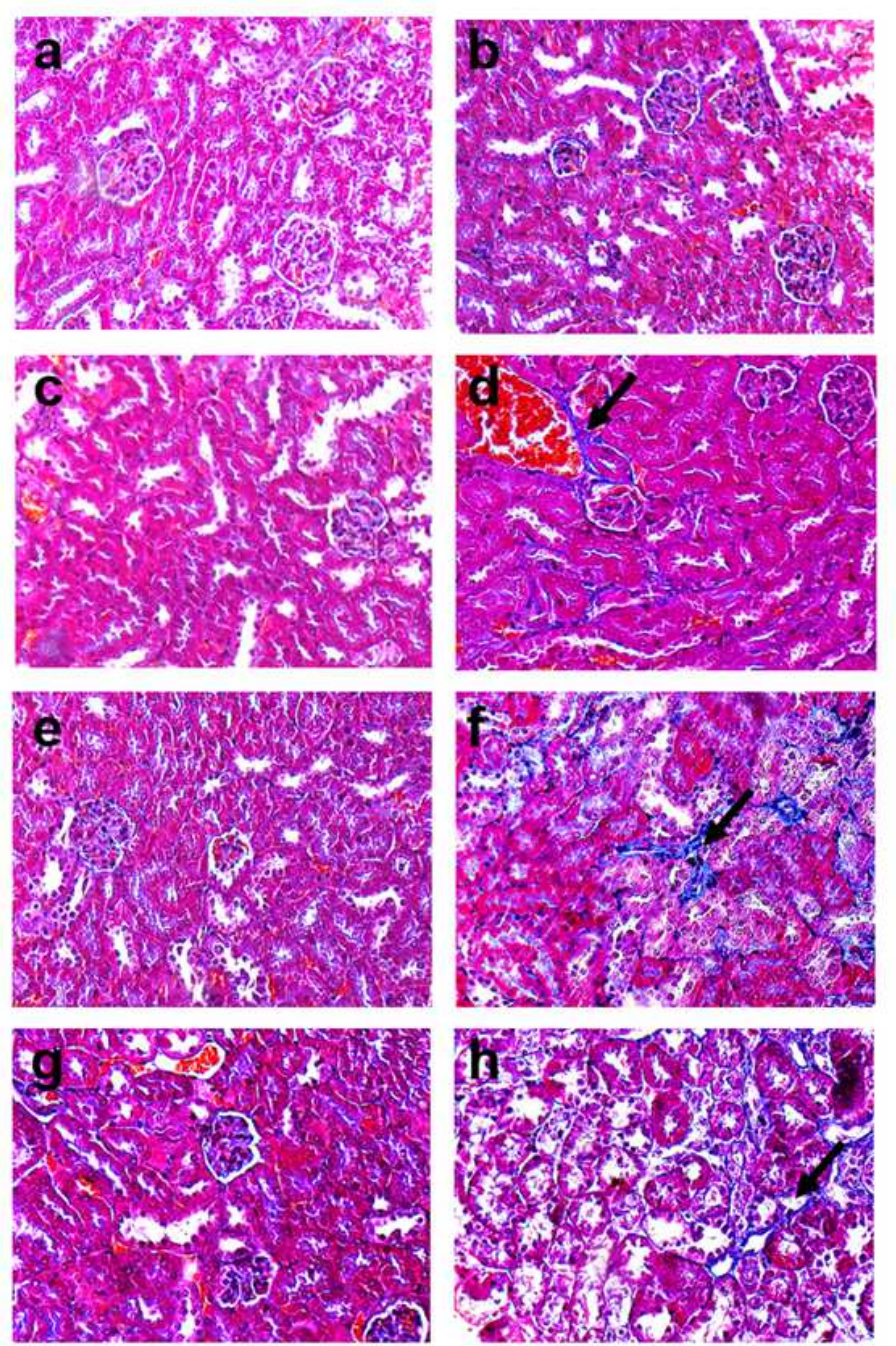
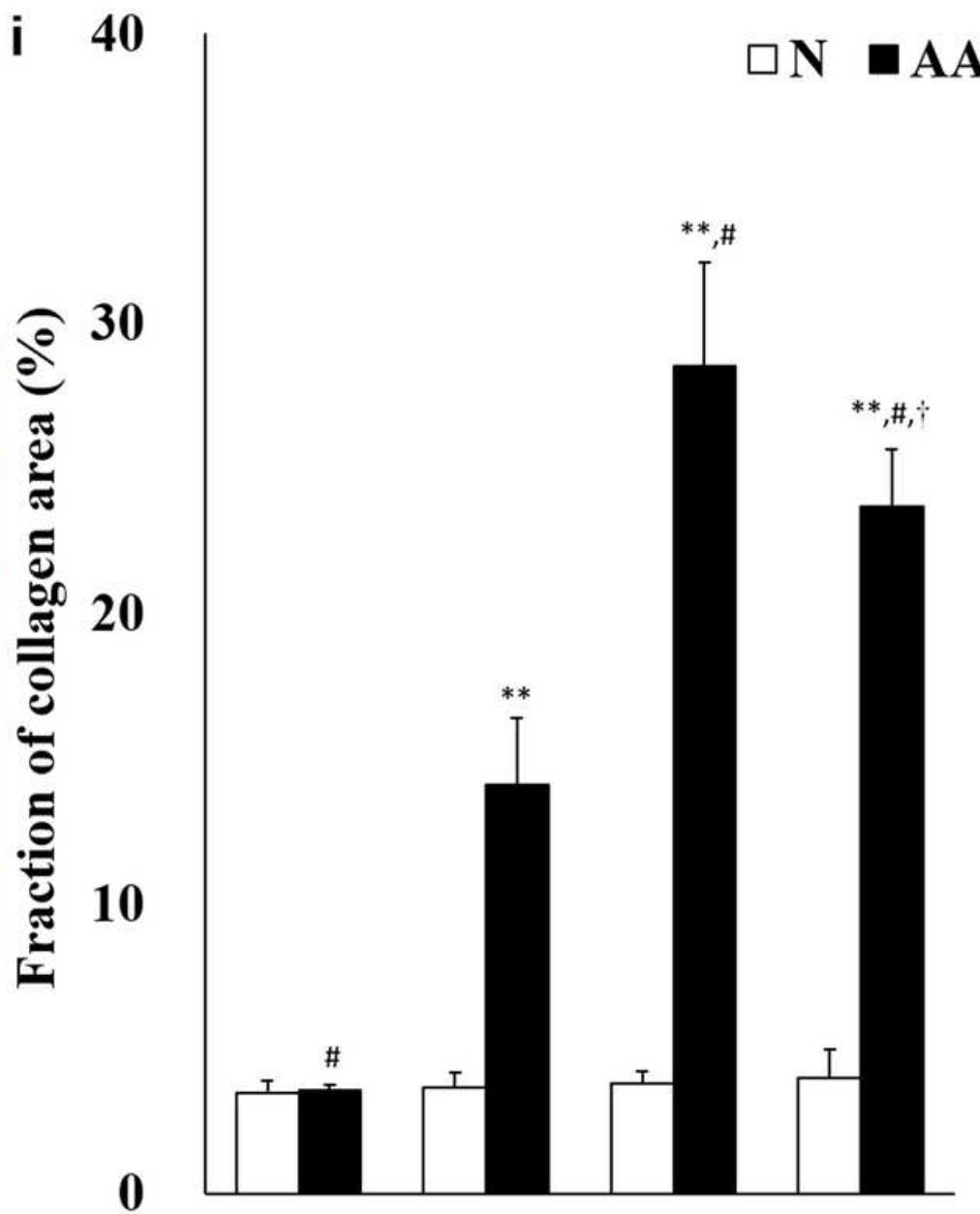

Day 28 Day 56 Day 70 Day 84

\section{Figure 2}

Masson's trichrome stained kidney sections and semi quantitative analysis of collagen deposition The fraction of interstitial fibrosis was assessed as the percentage of aniline blue stained area. (a-i) Representative images of Masson's trichrome stained kidney sections from the $\mathrm{N}$ group on day 28 (a) 56 (c), day 70 (e), and day 84 (g) and AA group on day 28 (b) 56 (d), day 70 (f), and day 84 (h). The arrow indicates collagen deposition. (i) Semiquantitative analysis of collagen deposition. $\mathrm{N}$ group , normal group; AA group, aristolochic acid group. ${ }^{\star *} \mathrm{p}<0.01$ vs. $\mathrm{N}$ group at the same time point, Student's t test; \# $p<0.05$ vs. AA group on day $56 p<0.05$ vs. AA group on day 70, repeated measures ANOVA. 


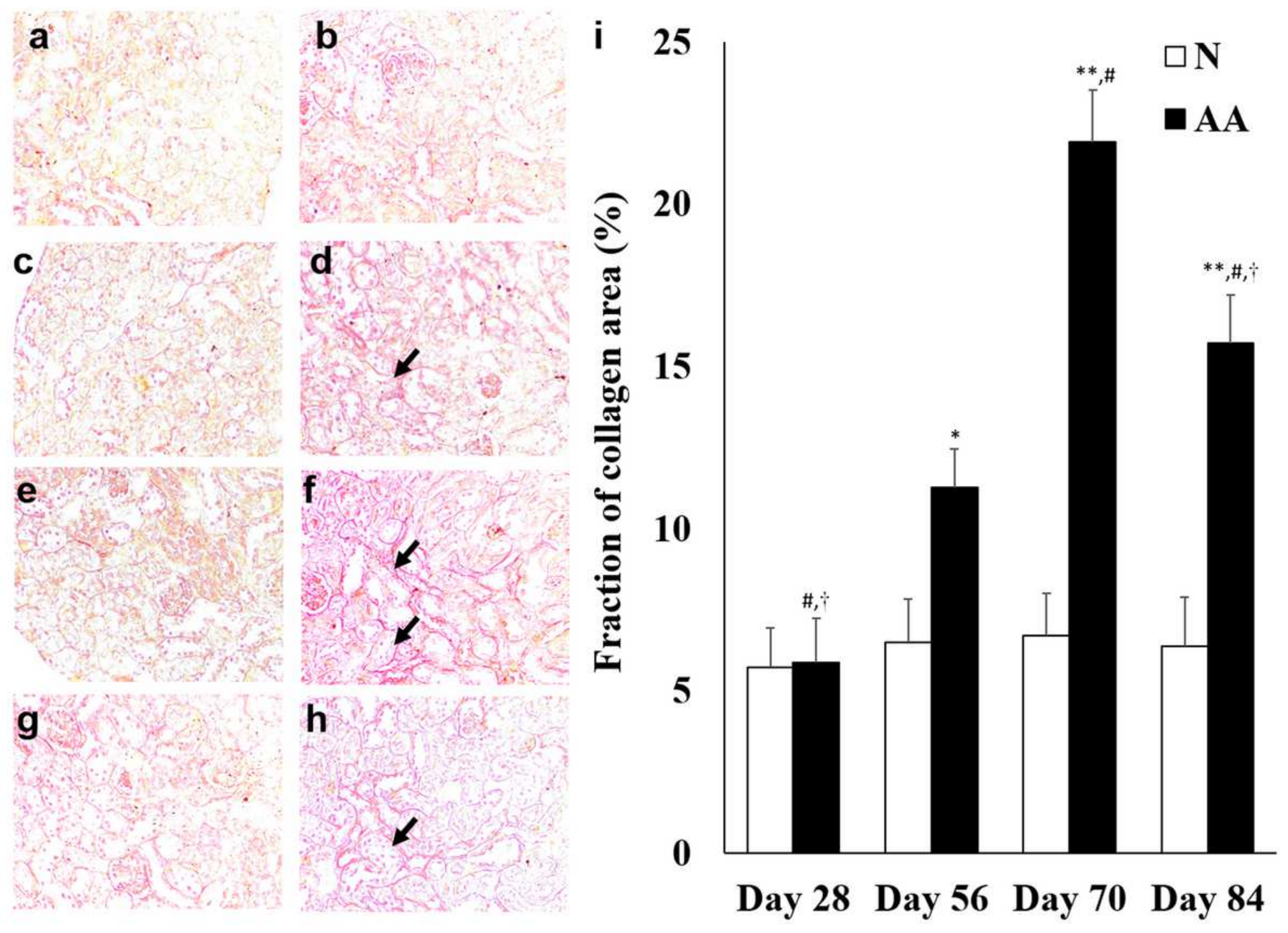

Figure 3

Picro Sirius Red stained kidney sections and semi quantitative analysis of collagen deposition The fraction of interstitial fibrosis was assessed as the percentage of red stained area. (a-f) Representative images of Picro Sirius Red stained kidney sectio ns from the N group on day 28 (a), 56 (c), day 70 (e), and day 84 (g) and AA group on day 28(b), 56 (d), day 70 (f), and day 84 (h). The arrow indicates collagen deposition. (i) Semiquantitative analysis of collagen deposition. $\mathrm{N}$ group , normal group; AA group, aristolochic acid group. ${ }^{*} p<0.05,{ }^{*} p<0.01$ vs. N group at the same time point, Student's t test; $\# p<$ 0.05 vs. AA group on day 56. $p<0.05$ vs. AA group on day 70, repeated measures ANOVA. 


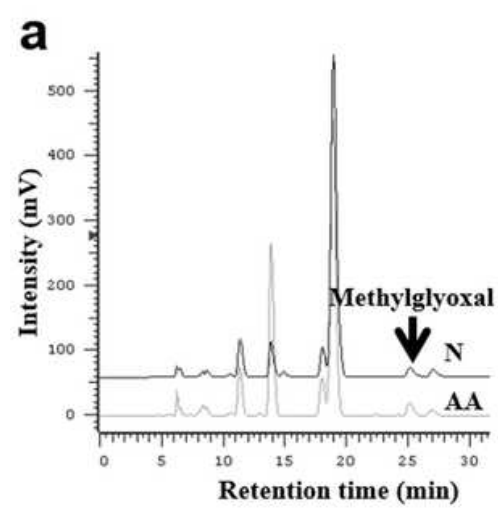

e

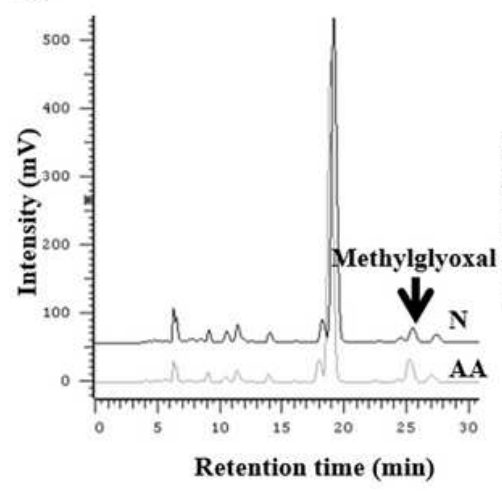

Retention time (min)

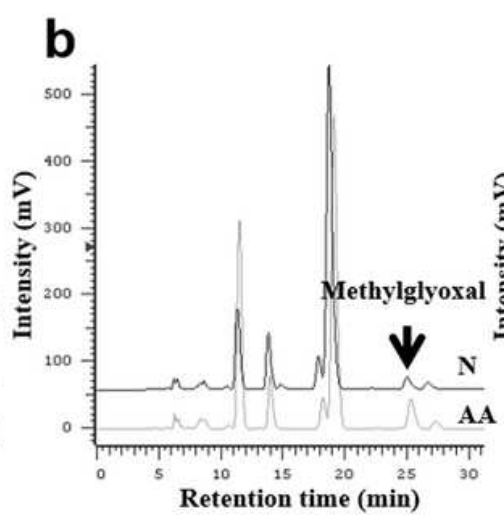

f

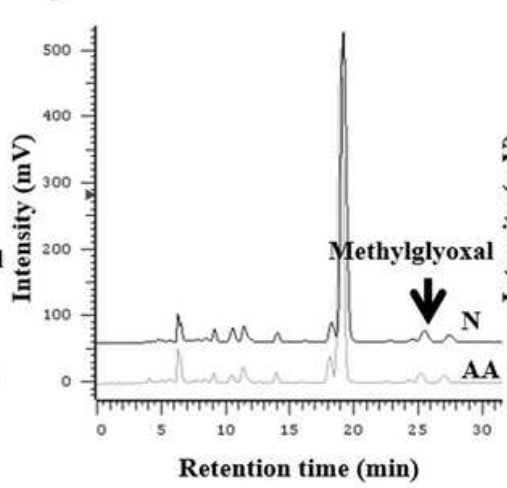

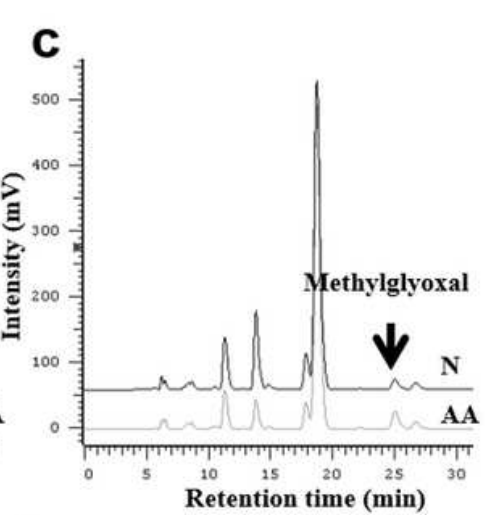

f

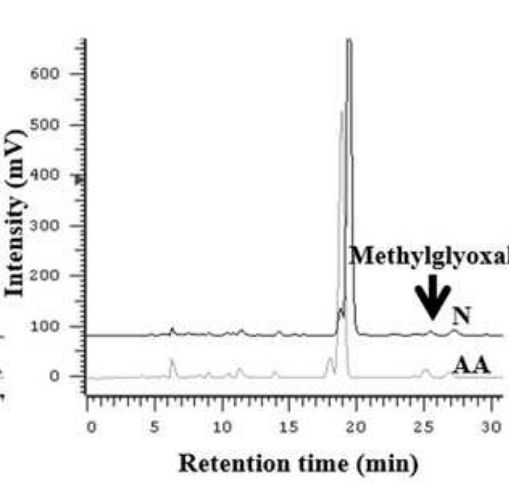

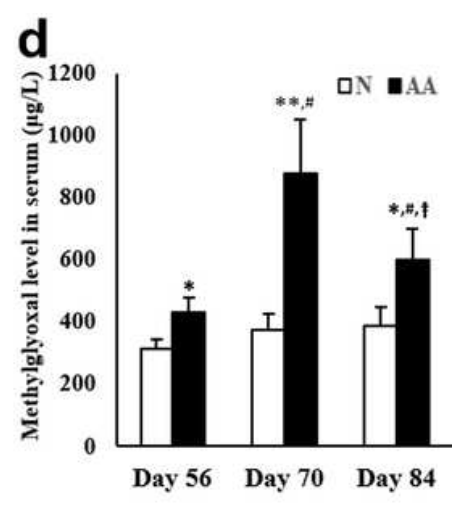

h

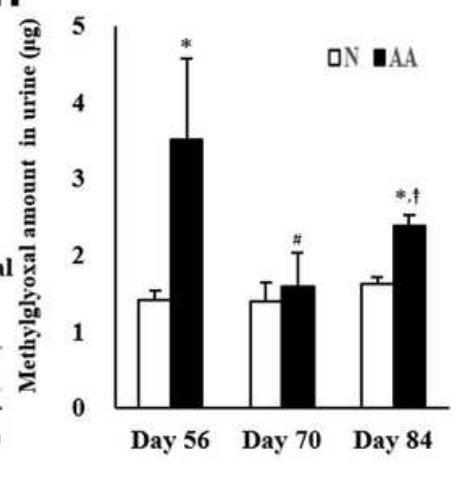

Figure 4

Chromatograms of analysis and contents of methylglyoxal in serum and urine HPLC chromatograms for serum methylglyoxal level on day 56 (a), day 70 (b), and day 84 (c). The corresponding peaks of methylglyoxal derivative was I abeled in HPLC chromatograms. (d) Serum methylglyoxal content. The white bars indicate the $\mathrm{N}$ group and the black bars indicate the AA group. $\mathrm{N}$ group, normal group; AA group, aristolochic acid group. HPLC chromatograms for urinary methylglyoxal on day 56 (e), day 70 (f), and day $84(\mathrm{~g})$. The corresponding peaks of methylglyoxal derivative was I abeled in HPLC chromatograms. (h) Urinary methy Iglyoxal content, calculated as methylglyoxal level $\times 12 \mathrm{~h}$ urinary volume. $\mathrm{N}$ group , normal group; AA group, aristolochic acid group. ${ }^{*} p<0.05$ and ${ }^{* \star} p<0.01$ vs. N group at the same time point, Student's t test; $\# p<0.05$ vs. AA group on day $56+p 0.05$ vs. AA group on day 70, repeated measures ANOVA. 

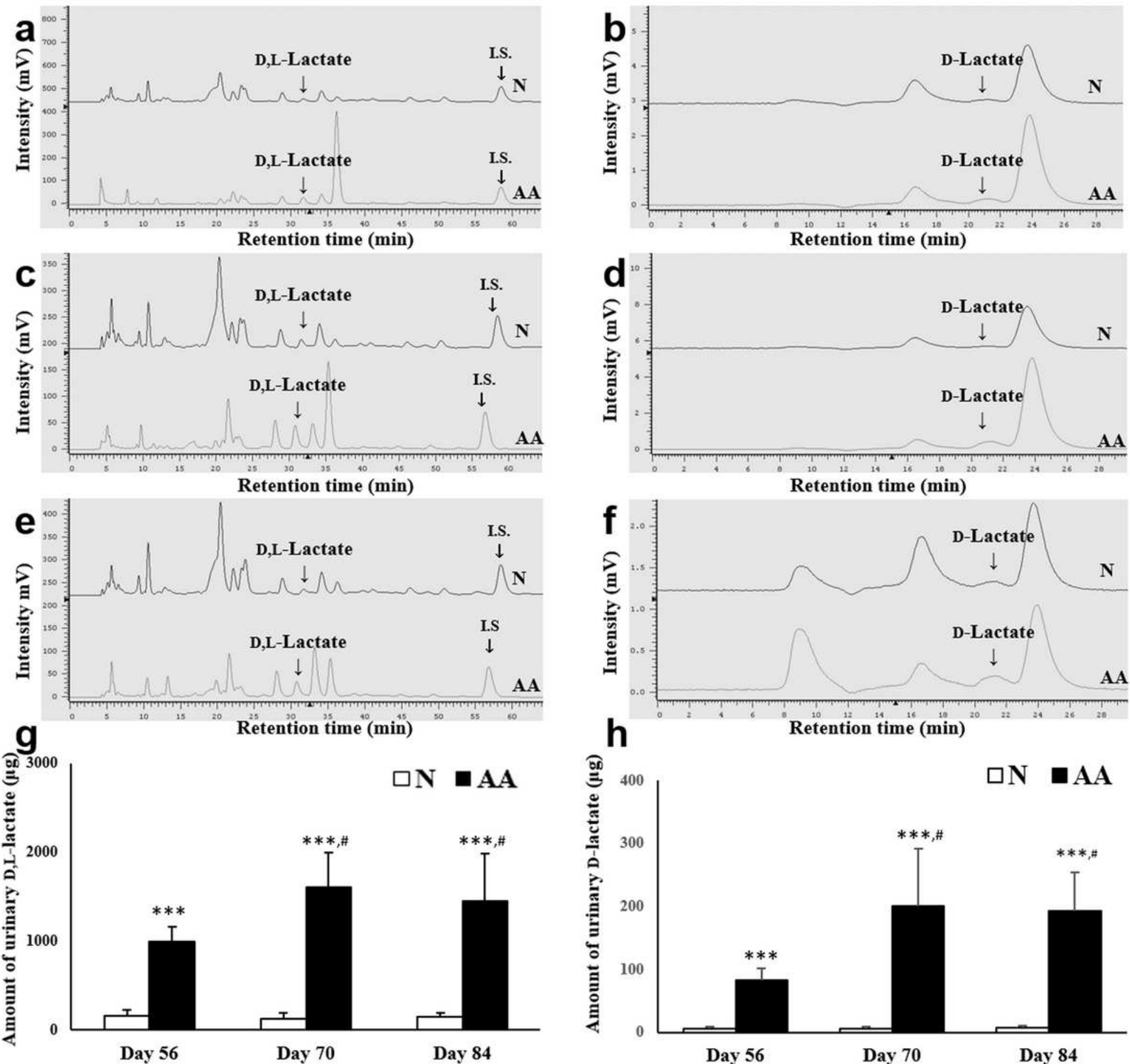

Figure 5

Chromatographs of analysis and contents of lactate ( D,L lactate and D lactate) in urine (a, c, e) HPLC chromatographs for the D,L lactate on day 56 (a), day 70 (c), and day 84 (e). (b, d, f) HPL C chromatographs for the $D$ lactate on day 56 (a), day 70 (b), and day 84 (c). The corresponding peaks of D,L lactate and D lactate derivative and internal standard (I.S.) was labeled in HPLC chromatograms. (g, h) Quantification of D,L lactate (g) and D-lactate (h) amount. Urinary D,L lactate amount, calculated as $D, L$ lactate level $\times 12 \mathrm{~h}$ urinary volume; urinary $D$ lactate amount, calculated as $D$ lactate level $\times 12 \mathrm{~h}$

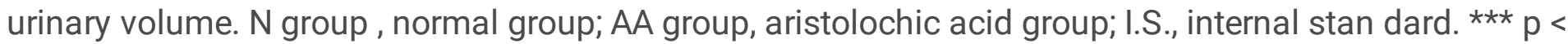


0.001 vs. N group at the same time point, Student's t test; $\# p<0.05$ vs. the AA group on day 56 , repeated measures ANOVA.

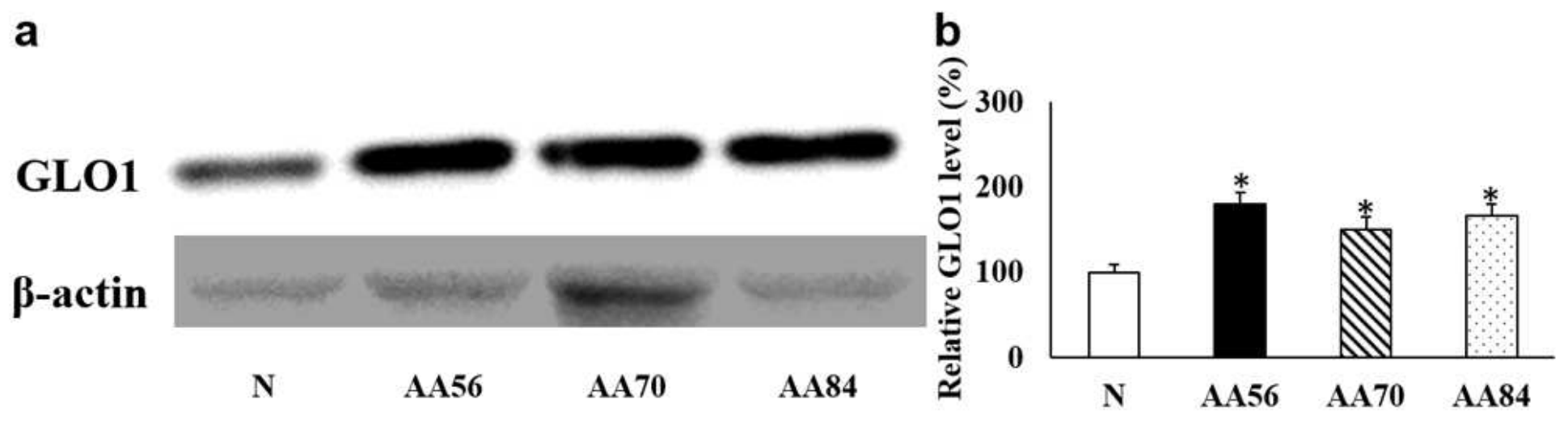

Figure 6

Western blot analysis and relative levels of glyoxalase 1(GLO1) in the kidney homogenates (a) Representative western blot of GLO 1; $\beta$ actin was used as an internal control. (b) Semi quantitative analysis of GL01 expression. AA56, AA group on day 56; AA70, AA group on day 70; AA84, AA group on day 84; $\mathrm{N}$ group, normal group; AA group, aristolochic acid group * $p 0.05$ vs. $\mathrm{N}$ group, ANOVA. 